\title{
Desafíos del desarrollo rural en Argentina: una lectura desde un territorio de la pampa húmeda
}

\section{Rural development challenges in Argentina: a reading from a Humid Pampa territory}

\author{
Silvia GorensteIn \\ Martín NAPAL \\ ANDREA BARBERO*
}

\begin{abstract}
In this paper we offer a critical reflection of the ideas that shape public actions to support small and medium rural farming producers, as well as the development and sustainability of their populations. We start from recognising the structural limits of these territories and discuss the current policies; in particular we address the proposal of new guidelines based on the Desarrollo Territorial Rural (Rural Territorial Development). We propose an analytical reading to set a series of challenges for the possibilities and policies towards rural development in the province of Buenos Aires, Argentina.
\end{abstract}

Keywords: rural development, Buenos Aires rural farming territory, rural farming policies, family agriculture, small livestock and farming producer.

\section{Resumen}

En este artículo se ofrece una reflexión crítica acerca de las visiones que moldean las acciones públicas en apoyo a los pequeños y medianos productores agrorrurales, y al desarrollo o sostenimiento de su población. Se parte del reconocimiento de los límites estructurales de estos territorios y se discuten las políticas vigentes; particularmente la propuesta de nuevos lineamientos encuadrada en el enfoque del Desarrollo Territorial Rural. Se propone una lectura analítica para plantear una serie de desafíos en torno a las posibilidades y políticas tendentes al desarrollo rural en la provincia de Buenos Aires.

Palabras clave: desarrollo rural, territorio agrorrural bonaerense, políticas agrorrurales, agricultura familiar, pequeño productor agropecuario.

* Universidad Nacional del Sur, Argentina. Correos-e: sgoren@criba.edu.ar, mjnapal@yahoo.com.ar, abarbero@criba.edu.ar. 


\section{Introducción}

Las nuevas dinámicas en la agricultura y, más ampliamente, en el sector agroalimentario, impactan tanto en las condiciones de producción de los pequeños y medianos productores como en las economías de las comunidades rurales, pueblos y pequeñas ciudades donde habitan estos sectores.

El objetivo del presente artículo es hacer una reflexión crítica sobre las visiones que moldean las acciones públicas para apoyar a estos estratos sociales agrarios y al desarrollo o sostenimiento de la población en áreas agrorrurales. Partiendo del reconocimiento de los limitantes estructurales que se manifiestan en este tipo de territorios, ${ }^{1}$ se discuten las políticas vigentes y la propuesta de nuevos lineamientos encuadrada en el enfoque del desarrollo territorial rural (DTR).

En tal sentido, es interesante comenzar rescatando las observaciones que formula Miren Etxezarreta cuando analiza el tema en el contexto europeo: "Es a partir de mediados de los ochenta que se acepta que la modernización agraria no sólo no puede resolver el problema del empleo rural ni de la equiparación de rentas de los agricultores, sino que lo empeora. El desarrollo rural ya no es la consecuencia de la organización social y espacial de la producción agraria [...] El desarrollo rural no surge espontáneamente de la organización productiva, se ha convertido en una opción social" (las cursivas son nuestras) (2003: 3). ${ }^{2}$

Estas reflexiones traducen muy bien las preocupaciones que dan sustento a las reformulaciones del paradigma del desarrollo rural (DR) durante la segunda posguerra. Pero, más importante aún, pone el acento en dos elementos clave a la hora de perfilar una nueva visión estratégica para el desarrollo rural. Por un lado, la cuestión de los condicionantes asociados a las lógicas globales-sistémicas que configuran el nuevo modelo agrícola y agroalimentario. Por otro lado, la necesidad de definir socialmente el alcance y contenido del mismo. Dicho de otro modo, las problemáticas rurales trascienden las cuestiones vinculadas con el cómo y qué se produce, el cuánto se diversifica o, entre otros, cuáles y cuántos empleos no rurales se generan; supone discutir cuál es

\footnotetext{
${ }^{1}$ La unidad territorial que integra la pampa húmeda argentina es lo que configura el medio rural de la provincia de Buenos Aires.

${ }^{2}$ En este mismo documento la autora contextualiza el proceso, planteando que hasta esa etapa la crisis de la agricultura y el medio rural se había resuelto con la emigración a las áreas industriales, pero desde los setenta, con la crisis y reconversión de la industria fordista, se agota esta forma de absorción de la emigración rural.
} 
el grado de DR que se pretende socialmente y, en la medida que se trata de ir moldeando paulatinamente este objetivo social, indagar en el modelo que se desea, así como la viabilidad, alcance y costo de la fórmula elegida. En concreto, la legitimización social del DR involucra un proceso de construcción de consensos políticos y sociales.

Desde esta perspectiva, en este artículo se examinan diferentes ángulos de la economía agraria y rural bonaerense -que forma parte de la región pampeana argentina (figura I)-, combinando la información empírica a través de un recorrido que trata de vincular las lógicas que interactúan e inciden en los sectores sociales más desprotegidos. Con esta descripción, se dará cuenta de las políticas y programas destinados al desarrollo rural de la provincia, poniendo énfasis en aquellas cuestiones que refieren a las orientaciones más generales destinadas al sector y su impacto concreto en la realidad que intenta modificar. Asimismo, se realiza una lectura crítica de las políticas que se formulan sobre la base del enfoque del desarrollo territorial rural. Por último, se proponen una serie de desafíos en torno a las posibilidades y políticas tendentes al desarrollo rural en la provincia de Buenos Aires.

\section{Rasgos estructurales del escenario agrorrural bonaerense}

La realidad bonaerense, ${ }^{3}$ y pampeana en general, plantea condiciones que se alejan bastante de la ruralidad campesina latinoamericana y de las economías locales poco integradas, con un amplio sector de empresas informales, muestra de buena parte de la evidencia empírica de los estudios de caso en América Latina. Como señalan Tsakoumagkos et al.: "No se trata de una región con base campesina, lo cual no significa que no haya pequeños productores. Tales productores existen pero presentan caracteres intersticiales o marginales y, comparados con los de otras regiones, muestran siempre mayor vinculación a los mercados y cuentan con tierra cuyo precio de mercado y su valor de renta les abren posibilidades con las que no cuentan pequeños productores de otras regiones" (2000: 19).

Estos sectores sociales son los que históricamente construyeron la territorialidad agrorrural bonaerense y demostraron "flexibilidad y eficiencia" (Lattuada, et al., 2006: 167) para adaptar-

\footnotetext{
${ }^{3}$ Este término se utiliza para hacer referencia a habitantes, condiciones y/o características de la provincia de Buenos Aires.
} 


\section{Figura I}

Región pampeana, provincia de Buenos Aires

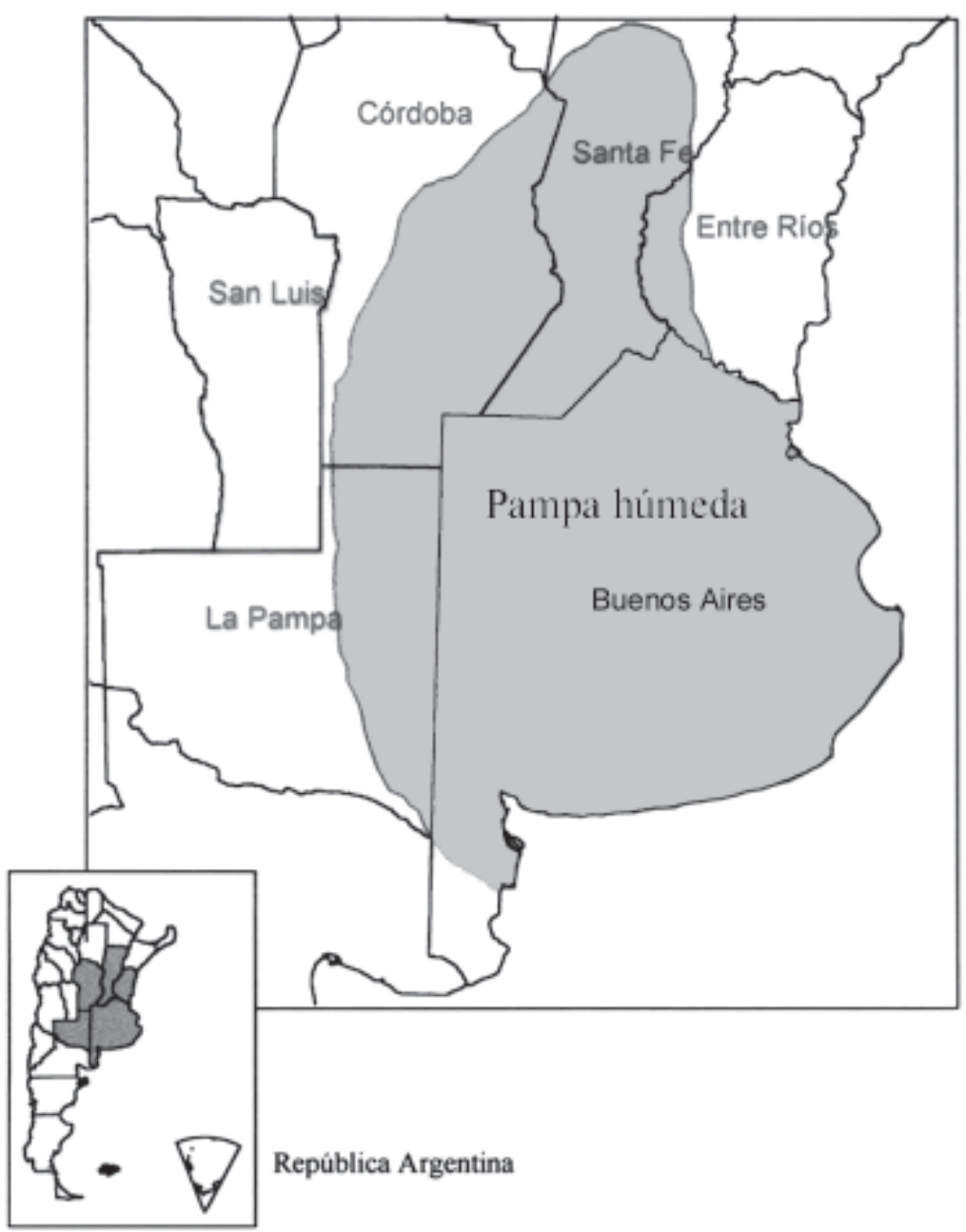

Fuente: IICA-PROINDER (2006).

se a los diferentes esquemas de acumulación. No obstante, frente a los cambios tecnológicos y organizacionales en la agricultura pampeana durante las últimas décadas, enfrentan el riesgo de perder su condición de productor, además del desplazamiento de su hábitat y el incremento de la pobreza familiar.

En primer lugar, existen factores y elementos muy rígidos y fuertemente enraizados en las lógicas y dinámicas de acumulación de los complejos agroalimentarios (CAA) que constituyen una de las bases centrales de este proceso de desplazamiento y de 
despoblamiento rural. Tanto en los caA de commodities tradicionales (cereales, oleaginosas y carne), como en aquellos de producciones intensivas o no tradicionales, se profundiza la concentración y centralización del capital, con nodos estratégicos transnacionalizados (proveedores de insumos y tecnologías, traders globales, etc.), y se difunden innovaciones (técnicas y organizacionales) que elevan las barreras de entrada para los pequeños y medianos productores familiares.

En segundo lugar, se plantean obstáculos a la inserción en mercados locales de trabajo, lo cual condiciona las posibilidades de acceder a fuentes de ingreso alternativas a la derivada de las explotaciones agropecuarias. La falta de nuevas oportunidades de empleo no agrícola, combinada con una serie de cambios que afectan el nivel de ocupación (familiar y salarial) del agro de la zona, constituyen las problemáticas más visibles de las localidades o pueblos más pequeños de la provincia, que atraviesan un proceso de crisis y vaciamiento poblacional. De este modo, en vastas zonas agrorrurales bonaerenses se plantea una especie de círculo vicioso: estructura económica fuertemente asociada a la actividad agropecuaria y ausencia de atractivos para nuevos proyectos y diversificación productiva, falta de oportunidades de empleo y expulsión o deterioro de los recursos humanos localizados (descalificación progresiva de la mano de obra, distanciamiento tecnológico de los agentes productivos, envejecimiento poblacional, etc.). Lo que ocasiona, entre otros efectos, deseconomías de aglomeración y complementación.

\section{Características de la pequeña producción agropecuaria bonaerense}

El Censo Agropecuario de 2002 registra en la provincia 51,058 explotaciones agropecuarias (EAP) con una superficie total de 25’787,364 ha, es decir, hubo una disminución relativa de más de 20,000 EAP y de casi un millón y medio de hectáreas respecto al censo de 1988. La mayor caída en el número de EAP se registró entre las que no alcanzan las 50 ha, seguida en orden decreciente por las que se ubican en los dos estratos siguientes, inferiores a 1,000 ha. Las mayores de este tamaño, en cambio, prácticamente se mantienen en número y aumentan casi $8 \%$ la superficie que controlan. 
Si medimos el fenómeno de la agricultura familiar según la metodología del PROINDER-IICA (2006), ${ }^{4}$ los sectores de la pequeña producción familiar bonaerense representaron, en 2002, algo más de 50\% de las explotaciones registradas. Un poco más de 27,000 EAP con una superficie media de casi 150 ha, así como una superficie de unas cuatro millones de ha (cuadro 1). En el cuadro 2 se registra el peso relativo de los tres tipos de la PPA: un poco más de $40 \%$ corresponden a PPA del tipo 1 , y con respecto a la superficie total, también tienen mayor peso individual los establecimientos tipo 1 (67.4\%), más del doble de la superficie ocupada por las EAP tipos 2 y 3 juntas. $^{5}$

En cuanto a su inserción productiva, los PPA explicaban un $14 \%$ del total de la superficie dedicada a la agricultura extensivas (cereales, oleaginosas y cultivos forrajeros), donde se destaca la presencia de los productores de mayor capitalización relativa $\left(\mathrm{PPA}_{1}\right)$. Estos datos censales no deberían, sin embargo, sobredimensionarse. Estudios más recientes revelan que los procesos de cesión de tierras entre los estratos de la PPA ocupan un papel importante. En este sentido, Daniel Slutzky (2003) observa que una parte significativa de la superficie incorporada por las grandes explotaciones proviene del alquiler de tierras antes trabajadas por pequeños y medianos productores.

En los cultivos intensivos (horticultura, floricultura, etc.) destaca la presencia de pequeños productores tipo 2 , mientras que la horticultura familiar capitalizada (PPA), con otras limitaciones y requerimientos, se ubica sobre todo en las producciones con

${ }^{4}$ Para los propósitos de este trabajo se considera el reprocesamiento de la información del Censo Agropecuario del 2002 realizada por el PRONDER-IICA (2006) donde, partiendo de las características de la explotación agropecuaria que dirigen, se contemplan los criterios más generales que engloban la pertenencia a este estrato productivo: 1) el productor trabaja directamente la explotación; 2) no emplea trabajadores no familiares remunerados permanentes; 3) no tiene como forma jurídica la sociedad anónima o comandita por acciones y 4) tienen una superficie total de hasta 1,000 ha y una superficie cultivada de hasta 500 ha o 500 unidades ganaderas (provincia de Buenos Aires). A partir de esta combinación de criterios, se distingue al conjunto de pequeños productores (PPA) en tres subtipos según su nivel de capitalización. Los del tipo 1 son los pequeños productores más capitalizados y con los recursos que poseen pueden acceder a la producción ampliada; los de tipo 2 son aquellos que tienen limitaciones para la producción ampliada o evolución económica de su explotación; los de tipo 3 manifiestan condiciones de inviabilidad económica y se mantienen en el campo por ingresos extraprediales y/o apoyo de programas públicos.

${ }^{5}$ Considerando el indicador de jornales equivalente al interior de la tipología, calculado en el agregado de la región pampeana, también revela la primacía del trabajo familiar en los establecimientos tipo $1(53 \%)$ y la significativa diferencia en relación con el agregado nacional (18\%), donde son las categorías más bajas (2 y 3) las que detentan la mayor parte del trabajo familiar (PROINDER-IICA, 2006). 


\section{Cuadro 1}

Indicadores de la pequeña producción agropecuaria según grandes agregados territoriales (2002)

\begin{tabular}{|c|c|c|c|c|}
\hline & & EAP totales & EAP PPA & $\begin{array}{c}\text { PPA/total } \\
\text { (porcentaje) }\end{array}$ \\
\hline \multirow{8}{*}{$\begin{array}{l}\text { Provincia de } \\
\text { Buenos Aires }\end{array}$} & Número & & & \\
\hline & de EAP & 51,116 & 27,168 & 53 \\
\hline & Superficie (ha) & $25^{\prime} 788,670$ & 4’029,070 & 16 \\
\hline & Jornales & & & \\
\hline & $\begin{array}{l}\text { equivalentes } \\
\text { a trabajadores }\end{array}$ & & & \\
\hline & permanentes & $38^{\prime} 014,240$ & $12 ’ 487,200$ & 33 \\
\hline & Superficie & & & \\
\hline & media (ha) & 505 & 148 & 29 \\
\hline \multirow{7}{*}{$\begin{array}{l}\text { Región } \\
\text { pampeana }\end{array}$} & Número & & & \\
\hline & de EAP & 103,700 & 58,733 & 57 \\
\hline & Superficie (ha) & $44^{\prime} 800,000$ & $8^{\prime} 082,113$ & 18 \\
\hline & Jornales & & & \\
\hline & trabajadores & & & \\
\hline & permanentes & $76^{\prime} 645,120$ & $27^{\prime} 549,600$ & 36 \\
\hline & $\begin{array}{l}\text { Superficie } \\
\text { media (ha) }\end{array}$ & 441 & 137.6 & 31 \\
\hline \multirow[t]{5}{*}{ País } & Número de EAP & 333,533 & 218,868 & 66 \\
\hline & Superficie (ha) & $174 ’ 808,564$ & $23,519,642$ & 13 \\
\hline & $\begin{array}{l}\text { Jornales } \\
\text { equivalentes a } \\
\text { trabajadores }\end{array}$ & & & \\
\hline & $\begin{array}{l}\text { permanentes } \\
\text { Superficie }\end{array}$ & $244^{\prime} 214,560$ & $132 ’ 158,560$ & 54 \\
\hline & media (ha) & 524 & 107 & 20 \\
\hline
\end{tabular}

Fuente: Elaboración propia con base en CNA (2002) y PROINDER-IICA (2006).

\section{Cuadro 2}

Cantidad de pequeños productores y superficie operada según tipo

\begin{tabular}{lrrr}
\hline & \multicolumn{1}{c}{$\mathrm{PPA}_{1}$} & \multicolumn{1}{c}{$\mathrm{PPA}_{2}$} & \multicolumn{1}{c}{$\mathrm{PPA}_{3}$} \\
\hline Número de EAP & 11,375 & 9,175 & 6,618 \\
\% sobre total PP Pcia. & 41.9 & 33.8 & 24.3 \\
Superficie (ha) & $2{ }^{\prime} 714,576$ & 905,833 & 408,661 \\
\% sobre total PP Pcia. & 67.4 & 22.5 & 10.1 \\
Sup. media (ha/EAP) & 238.6 & 98.7 & 61.7 \\
\hline
\end{tabular}

Fuente: Elaboración propia en base en CNA (2002) y PROINDER-IICA (2006). 
mayor tecnología incorporada (invernáculos). ${ }^{6}$ Asimismo, los pequeños productores tipo 3 , caracterizados como estrato en el cual los programas de asistencia técnica y financiera han operado como soporte fundamental para su sobrevivencia, estarían ubicados, mayoritariamente, en el cultivo de aromáticas.

Como en el resto de la región pampeana, el número de personas ocupadas en la agricultura ha disminuido. Pero, además de esta caída cuantitativa, hay un deterioro cualitativo relacionado con las condiciones contractuales del trabajo rural. Tanto en las áreas de agricultura intensiva (fundamentalmente horticultura) como en las extensivas, se observa un progresivo crecimiento del trabajo precario e informal que responde, por un lado, a las nuevas dinámicas tecnoproductivas $\mathrm{y}$, por otro, a las lógicas de acumulación de los productores y núcleos organizadores de las diferentes tramas.

\section{Contexto y orientaciones de las políticas agrorrurales}

Desde fines de 2001 se instalaron nuevas condiciones macroeconómicas y sectoriales en el país. ${ }^{7}$ Entre otros efectos, se produce una fuerte recuperación de la competitividad del conjunto de bienes intercambiables; entre ellos y sobre todo, los agroalimentarios. Además de los commodities tradicionales (oleaginosas y cereales), surgieron nuevas ventajas en productos antes orientados al mercado interno.

En este contexto macroeconómico, la mayor parte de las acciones y programas se orientan al aumento de la competitividad en los mercados mundiales, consistente con el sesgo exodirigido $y$ neutral de la política sectorial. Esta situación refuerza la posición relativa de los grandes capitales que operan en la agricultura, quienes extienden su control sobre tierras cultivables, hasta

\footnotetext{
${ }^{6}$ Dentro de este grupo se plantearían situaciones diferentes en el marco de estrategias que Benencia y Quaranta (2005) denominan de expansión flexible: las empresas familiares, con asalariados y/o medieros, combina la tierra en propiedad con la toma de tierra en arrendamiento o bien la intensificación de la producción a través de la adopción del invernáculo.

${ }^{7}$ El modelo económico durante los noventa del siglo pasado se caracterizaba distintivamente por la paridad cambiaria fija uno a uno entre el peso argentino y el dólar estadounidense. El modelo colapsó, por un lado, por la incapacidad de incrementar las exportaciones (tipo de cambio alto, bajo precio internacional de las commoditties, entre otras) y por otro, por la imposibilidad de obtener crédito externo luego de las crisis económicas en los países emergentes (México, Corea, Rusia, Brasil). Lo anterior desembocó en casi tres años consecutivos de recesión económica en el periodo 19982001. Como consecuencia, en diciembre de 2001 el país se declaró en default y en enero de 2002 salió del régimen de convertibilidad.
} 
ahora, exentas de esta modalidad de explotación. ${ }^{8}$ Si bien la mejora en los precios relativos, la devaluación y el alza de precios internacionales ha favorecido la ecuación económica de explotaciones agropecuarias de pequeños y medianos productores, se puede conjeturar que este fenómeno no alcanza para frenar la tendencia estructural de desplazamiento (económico y tecnológico) que enfrentan estos sectores. En tal sentido, continúan las políticas sociales dirigidas a los actores rurales más vulnerables (PPA, trabajadores, familias).

\subsection{Los programas de desarrollo rural (PDR)}

Durante la década de los noventa del siglo xx, los programas orientados a la agricultura familiar y, más en general, a la pobreza rural se concibieron desde una perspectiva conceptual y política que enfatizaba las ineficiencias de la intervención directa del Estado en la planificación y asignación de recursos. En este marco, la idea de una intervención estatal selectiva y de bajo costo, dirigida a los grupos rurales más vulnerables, resultaba atractiva para articular cierta respuesta pública financiada, además, por organismos internacionales.

1) Programas focalizados. Estos programas siguen vigentes en el periodo actual. La información del cuadro 3, con indicadores de ejecución de los mismos en todo el país para el año 2005, revela que se ha asistido a unas 255,500 familias distribuidas entre PPA, trabajadores rurales y familias pobres de localidades de menos de 2,000 habitantes. Se plantea una diferenciación básica asociada a las situaciones sociales bajo la línea de pobreza. En efecto, del total de familias asistidas, más de dos tercios lo fueron a través del programa Prohuerta, destinado principalmente a la autoproducción y consumo de alimentos de familias rurales situadas bajo este límite. La cobertura del Programa Social Agropecuario (PSA) alcanzó unas 50,000 familias rurales pobres, mientras que el Programa Cambio Rural, orientado en general a grupos de PPA en mejor posición ( PPA $_{1}$ ) representa sólo 2\% del total de familias asistidas, aunque alcanza más de $16 \%$ de los grupos que recibieron ayuda.

El apoyo de estos programas se centró en asistencia técnica, sólo 2\% de las familias rurales asistidas -excluyendo al Prohuer-

\footnotetext{
${ }^{8}$ Para un análisis sobre las formas organizacionales y estrategias de expansión que se han difundido en las dos últimas décadas, veáse Basualdo y Khavisse (1993), Neiman y Lattuada (2005) y Teubal (2006).
} 


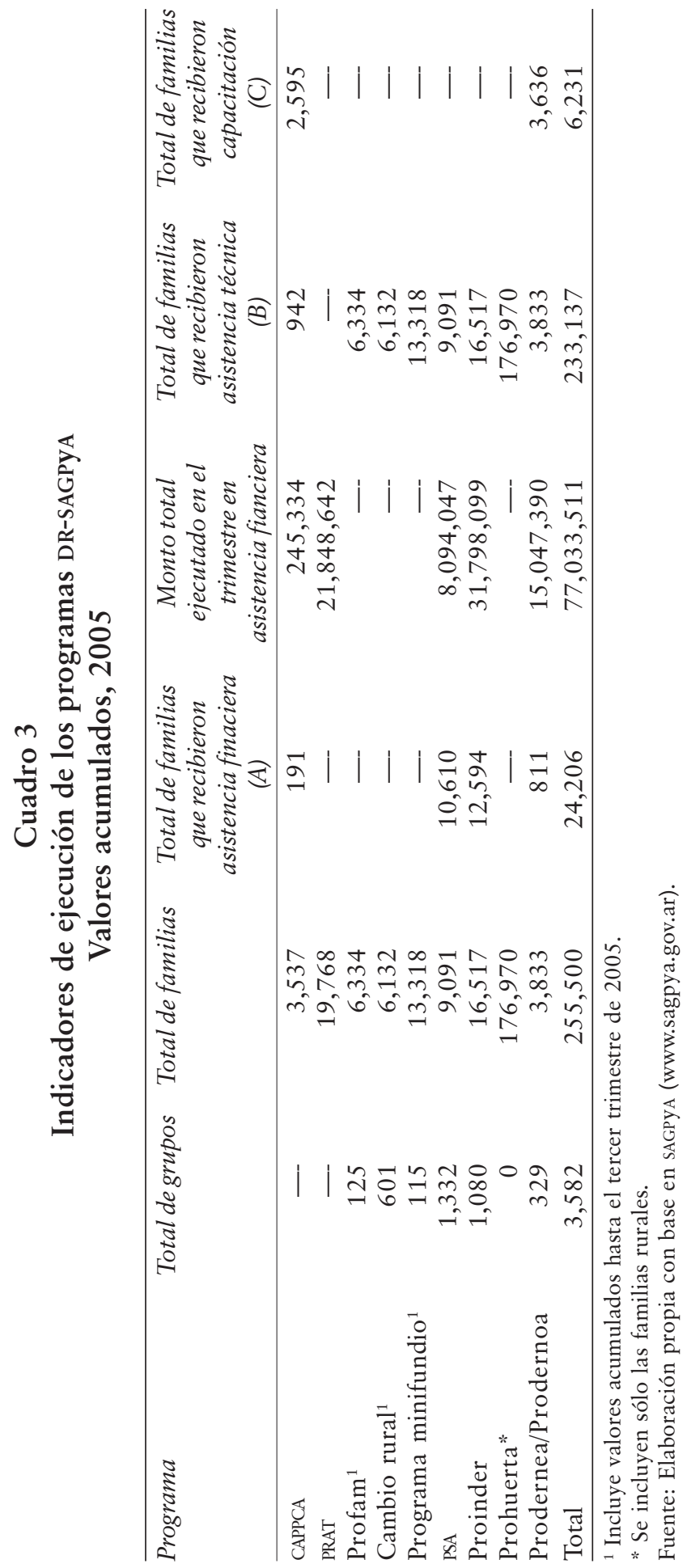


ta- recibieron capacitación. La ayuda financiera ${ }^{9}$ (9.5\%) ejecutada en el año 2005 alcanzó un promedio de \$3,182 por familia beneficiaria. ${ }^{10}$ Este valor medio sólo lo superaron los programas Prodernea/Prodernoa, ${ }^{11}$ donde se alcanzaron niveles cercanos a los veinte mil pesos, un capital operativo mucho más razonable desde el punto de vista económico y menos distante desde el punto de vista del desarrollo en general, al menos si se compara con los escasos \$ 763 que alcanzó la provisión financiera promedio del PSA. ${ }^{12}$

Si estas cifras se comparan con indicadores sectoriales clave, como el valor de las exportaciones y de las retenciones, la escasa importancia de estas respuestas públicas se hace más evidente: la suma de la asistencia financiera, provista desde los PDR, sólo representa poco más de tres milésimas partes de las exportaciones primarias de Argentina y $2 \%$ de las retenciones a las exportaciones primarias en ese mismo año.

La ejecución de los PDR nacionales es descentralizada. La provincia de Buenos Aires, más allá de la dimensión de su participación en este financiamiento nacional, posee un área de atención específica al desarrollo rural y ha operado sus propios programas Prohuerta -huertas bonaerenses- y Cambio Rural. Este último cuenta con sus propios técnicos para asesorar a los grupos de productores y se articula (explícita o implícitamente) con las áreas de extensión del INTA) ${ }^{13}$ asimismo, a través de las unidades operativas de extensión (Chacras) se desarrollan otros programas de capacitación y asistencia técnica.

2) Promoción de producciones intensivas. La promoción de producciones intensivas (conejos, cerdos, apicultura, arándanos, entre otros) lleva más de una década y sus resultados se pueden extrapolar a un gran número de experiencias en comunidades locales bonaerenses. Estos programas se concibieron como elementos complementarios de las estrategias de desarrollo local (rural y/o urbano), apoyando proyectos con bajos requerimien-

\footnotetext{
${ }^{9}$ En la mayoría de los programas la asistencia financiera toma la forma de subsidio no reintegrable.

${ }^{10} \mathrm{La}$ asistencia financiera promedio fue levemente superior, a valor dólar, en el periodo 1999-2001 (\$/dólar 1.361) y bajó durante los dos primeros años después de la devaluación (\$1.120).

${ }^{11}$ Programas destinados al noreste (provincias de Misiones, Corrientes, Chaco y Formosa) y al noroeste (Jujuy, Salta, Tucumán y Catamarca).

${ }^{12} \mathrm{La}$ cifra surge al promediar todos los ítems de asistencia financiera contemplados en el programa.

${ }^{13}$ El Instituto Nacional de Tecnología Agropecuaria es el que históricamente se ha encargado de la asistencia y transferencia de tecnología al sector agropecuario de Argentina, y en particular de la provincia de Buenos Aires.
} 
tos de capital (tierra, equipamiento, etc.) que, más o menos rápidamente, permiten generar ingresos alternativos a los beneficiarios. Al mismo tiempo, como son actividades comparativamente intensivas en mano de obra, garantizan la absorción de fuerza de trabajo familiar disponible (hijos, familiares sin remuneración) con un costo de oportunidad casi nulo, dadas las escasas opciones de empleo (parcial o total) en sus entornos suburbanos

En términos generales, los programas que promocionan pequeñas producciones intensivas o no tradicionales, concebidas como espacios de inserción competitiva de la agricultura familiar, se caracterizan por:

- Una visión productivista que opaca la perspectiva de corto, mediano y largo plazos asociada a los mercados existentes o potenciales, fluctuaciones de precios, márgenes y otros elementos que hacen que los proyectos promocionados sean rentables y sustentables económicamente. ${ }^{14}$

- Las acciones se centran en el eslabón primario ignorando la lógica global (sectorial) de la trama o CAA. Se interviene, entonces, donde las barreras de entrada suelen ser bajas pero sin atenuar las dificultades para alcanzar la unidad económica mínima.

- No actúan sobre los nodos concentradores, desconociendo que las fuertes limitaciones para que se produzca la incorporación de la agricultura familiar como proveedora regular (de la agroindustria, de la gran distribución minorista, frigoríficos, etc.) requiere una política de incentivos específicos por parte del sector público. Aun en el segmento de los pequeños productores con mayor capacidad empresarial es necesario un arbitraje para amortiguar los costos de transacción implicados en la participación, supervisión y coordinación de muchos pequeños proveedores. ${ }^{15}$

\subsection{Otras politicas públicas que inciden en el desarrollo rural}

Otro conjunto de programas que actúan sobre actores y/o territorios locales se articulan con las políticas agrorrurales pro-

\footnotetext{
${ }^{14}$ Esta lógica de intervención, sin una clara definición del mercado objetivo, puede inducir proyectos y un caudal de oferta que rápidamente enfrentan sus límites, como sucede en el caso de la cunicultura.

${ }^{15}$ Este factor, tal como se visualiza en diversos estudios, hace que los grandes compradores prefieran grupos reducidos de proveedores, en general, medianos y grandes.
} 
vinciales y nacionales. Si bien son unos cuantos (Manos a la Obra, Volver, Pueblos, Trabajo Dignifica, etc.), es posible identificar las grandes orientaciones y criterios de intervención que los rodean. A grandes rasgos se pueden delinear los siguientes ejes.

- La población meta se constituye por los sectores sociales más vulnerables (familias pobres, pequeñas localidades con elevados niveles de pobreza, desempleados, microemprendedores, etcétera).

- Otorgan subsidio para ejecutar, generalmente en el ámbito local, proyectos asociativos vinculados con el mejoramiento de la inserción comercial de micro y pequeños productores en cadenas de producciones intensivas o la implementación, asociada, de un eslabón de procesamiento de los productos primarios para agregarles valor (aquí se suele mencionar el caso de las extractoras comunitarias a cargo de cooperativas de PPA de miel).

- Utilizan metodologías participativas (diagnóstico, definición de objetivos y destinatarios de las propuestas, mesas de concertación local).

- Intervienen los municipios y gestores públicos territoriales contratados por las áreas ministeriales responsables. ${ }^{16}$

- Promueven nuevas actividades y fuentes de empleo, fomentando la cooperación y la articulación de redes socioterritoriales

- Proveen capacitación y asistencia técnica, inducción y fortalecimiento organizacional y pequeños financiamientos (subsidios directos o a través de compras de insumos y/o equipamiento).

En síntesis, los programas dirigidos a actores y territorios agrorrurales son unos cuantos, pero enfrentan fuertes desafíos para revertir o, de algún modo, atenuar los efectos de las dinámicas altamente concentradoras y excluyentes que operan desde las lógicas sectoriales de las cadenas productivas que impactan en estos territorios. Cabe hacer tres observaciones complementarias. Primera, la superposición de acciones que se traduce en falta de coordinación entre las áreas responsables, desarticulación institucional, competencias explícitas o implícitas entre los equipos que operan en el territorio y, en muchos casos, ejecucio-

${ }^{16}$ En el programa Pueblos, estos gestores los selecciona el gobierno y la comunidad local. 
nes en un periodo limitado y suspensión de las políticas. Segunda, la problemática relativa a las prácticas clientelares donde la captura del beneficiario se puede dar en el marco de una puja entre dos frentes (área respectiva del municipio versus la intermediación territorial propia de los programas). Tercera, los problemas derivados de los proyectos productivos promovidos en la medida que el apoyo otorgado no asegura los recursos estratégicos (tierra, tecnología, información, etc.) para mejorar la capacidad de reproducción y sustentabilidad económica dentro de las cadenas productivas.

\section{Los nuevos lineamientos: el enfoque del desarrollo territorial rural}

En las dos últimas décadas, distintos organismos internacionales coinciden en señalar nuevas propuestas para atenuar la pobreza rural. ${ }^{17}$ Desde el enfoque de desarrollo territorial rural se plantea la búsqueda de soluciones globales -no sectoriales- encuadradas en una serie de principios generales para la formulación de las nuevas políticas rurales (De Janvry y Sadoulet, 2001; BID, 2005). ${ }^{18}$

- La necesaria coordinación entre las políticas agrícolas y de desarrollo rural con las políticas macroeconómicas.

- La conexión de los pequeños y medianos productores a los sectores dinámicos de la agricultura, estimulando y apoyando la innovación en productos, servicios, procesos, organización o gestión.

- Impulso simultáneo a todas las dimensiones que componen el ingreso del habitante rural pobre. Las políticas dirigidas para la inclusión de los sectores pobres y socialmente excluidos deben contemplar, entre otros, la creación de empleos en actividades no agrarias ubicadas en zonas rurales, así como la implementación de programas especializados que apunten a potenciar sus capacidades y el acceso a activos productivos.

${ }^{17}$ Salama (2006) da cuenta de los grandes lineamientos que se desprenden de los organismos internacionales cuando describe la evolución desde los diez primeros mandamientos del Consenso de Washington para los objetivos del milenio, en los cuales se integra en primer lugar la reducción drástica de la pobreza (disminuir los valores absolutos existentes en 1990 a la mitad en 2015).

${ }^{18}$ Una interesante y oportuna reflexión crítica sobre el paradigma de DTR también se puede encontrar en: Manzanal (2006) y Abramovay (2006). 
- En todos los casos se debe tener en cuenta la complementariedad y adecuación del marco institucional para lograr la participación y canalización de las iniciativas locales, sociales y privadas. En esta línea, el enfoque pone el acento en: 1) el protagonismo de las organizaciones de la sociedad civil y los gobiernos locales; 2) la descentralización municipal como instrumento potencialmente efectivo para implementar planes y programas de desarrollo rural y 3) la reconstrucción y fortalecimiento de la institucionalidad rural sobre la base de diferentes organizaciones de la sociedad civil y la asociación público-privado.

Este enfoque ha comenzado a permear en el discurso y acción en torno al desarrollo rural en los países latinoamericanos. En Argentina, la propuesta para la estrategia de desarrollo rural (Rimisp, 2006) sigue estos lineamientos y se encuadra en la percepción de una nueva oportunidad -asociada al actual escenario sectorial favorable-, a fin de operar sobre los efectos perversos (sociales, ambientales, económicos, territoriales) acumulados en las últimas décadas tras el libre accionar de las lógicas del mercado. Vale la pena adentrarse en los contenidos de esta propuesta.

Las recomendaciones de política se basan en la concepción de competitividad territorial, como fenómeno sistémico, y de la institucionalidad rural, en tanto entramado de relaciones público-privadas o entre distintos agentes en una misma cadena de valor, promoviendo: 1) la vinculación competitiva de los territorios rurales con mercados más dinámicos, a través de procesos de transformación productiva y 2) coordinación y relaciones de cooperación entre los agentes económicos, sociales y gubernamentales, mediante procesos de desarrollo institucional. En tal sentido, se hace hincapié en la revisión de los criterios de focalización de los programas de desarrollo rural implementados hasta el momento ${ }^{19}$ y sugiere: políticas y proyectos entre sectores rurales pobres y otros, sociales y económicos, ubicados en segmentos de cierta capacidad y/o potencial económico; a su vez,

${ }^{19}$ Existe cierto consenso entre los especialistas respecto de las potencialidades de los programas existentes a la hora de pensar en un tejido institucional que acompañe y facilite el proceso de desarrollo rural. En términos generales, estas observaciones se nutren del análisis de experiencias en distintas regiones rescatando la importancia de la gestión institucional y de las metodologías participativas para inducir procesos de transformación y mejoramiento de las comunidades agrorrurales, en el marco del aprendizaje social, los técnicos formados, las relaciones de confianza y la organización de los beneficiarios de estos programas (Manzanal, 2005; Carballo, 2001; Soverna, 2004). 
pasar de los microproyectos locales a iniciativas socioeconómicas de mayor envergadura económica.

Las producciones intensivas aparecen como un área a priorizar dentro de estos lineamientos:

De existir una política de incentivos, la potencialidad competitiva de la agricultura familiar de producción debe buscarse en aquellos productos intensivos en trabajo [las cursivas son nuestras] y en supervisión, que carecen de economías de escala significativa en la fase primaria y que tienen un alto valor por hectárea y por unidad de peso (para que los costos de transporte no sean una limitante). Quedan por lo tanto fuera todos los commodities (cereales, granos y sus derivados) y otros rubros tradicionales (Informe final Rimisp, 2006).

No se contemplan, sin embargo, potenciales implicaciones a mediano plazo (al menos en alguna de estas producciones) derivadas de la incorporación de tecnologías ahorradoras de mano de obra (equipamiento informatizado en tareas poscosecha, maquinas recolectoras, etc.), así como otras dinámicas innovadoras que son más intensivas. Otra cuestión, no menor, es el tipo de trabajo rural que en este momento generan. En las cuencas de cultivos intensivos (frutas, hortícolas, etc.) se suelen definir relaciones y condiciones laborales que operan como una suerte de círculo vicioso; trabajo en negro, contrataciones intermediadas por figuras informales y sin las coberturas sociales mínimas, presión estacional sobre las infraestructuras básicas locales; crecimiento de enfermedades infecciosas, entre otras. Aquí hay cuestiones que hacen al marco regulatorio pero, a su vez, se plantean otras problemáticas asociadas al ámbito que compete a los gobiernos y las comunidades locales.

Tomando en cuenta los elementos señalados en estos lineamientos, a continuación hacemos una breve incursión crítica en torno a dos aspectos clave, a la vez específicos y complementarios, englobados en la formulación de propuestas orientadas a favorecer e impulsar la agricultura familiar y el desarrollo rural.

\subsection{Agricultura familiar e innovación}

Si bien no hay una propuesta detallada en torno a cómo promover dinámicas innovadoras en los sectores de la pequeña producción rural, se plantea que los esquemas de apoyo a la innovación tecnológica deben contemplar el aporte no reintegrable o financiamiento de riesgo a los proyectos de preinversión e inversión 
en todos los aspectos vinculados con la producción rural (producción, comercialización, organización). Se propone, a su vez, facilitar y garantizar los servicios profesionales especializados ${ }^{20}$ y sistemas de información y aprendizaje con base territorial. Estas consideraciones generales acompañan el énfasis especial del Rimisp en torno a la adaptación innovadora de los PPA ligados, fundamentalmente, a las tramas de producciones intensivas.

Rossi y León señalan que es necesario

programar e incentivar prioritariamente, aquellos desarrollos tecnológicos que pudieran volcarse para incrementar la rentabilidad de la pequeña producción, a través de las dos vías posibles: aumento de productividad de la actividad predominante y/o identificación de nuevos rubros con valores unitarios de producción más elevados [...] Una importante cantidad de hechos innovativos de las instituciones podrían ser canalizados y desarrollados comercialmente por la pequeña producción en estructuras asociativas que posibiliten lograr escalas adecuadas. Para que ello sea posible, no solamente hay que desenvolver este tipo de tecnologías en las instituciones, sino generar la preocupación e instrumentos necesarios para su posterior transferencia y adopción por parte de las pequeñas explotaciones agropecuarias (2005: 35-36).

Los problemas de adecuación tecnológica de los sectores de la pequeña producción agraria se entrelazan a un conjunto de rasgos básicos y comunes (escasa disponibilidad de tierra, condiciones ecológicas adversas, precios y condiciones de los mercados a los que pueden acceder, disponibilidad y costos de los insumos, entre otras) que trascienden la política tecnológica y de innovación.

En tal sentido, es oportuno mencionar las reflexiones de Graziano da Silva (1999: 63, 66, 135) cuando discute conceptual y políticamente la cuestión tecnológica para la agricultura familiar en Brasil. Un primer aspecto, plasmado en el hilo argumental de este autor, es de naturaleza estructural. En efecto, la generación de tecnologías adecuadas y su adopción por parte de los PPA no resolverían la restricción básica y fundamental: la apropiación de los frutos del aumento de la productividad que la modernización trae consigo. En otros términos, la adecuación tecnológica no garantiza que estos sectores puedan capturar una mayor apropiación del excedente producido porque, más temprano que tarde, la producción se debe realizar en mercados capitalistas. El segundo aspecto alude a las dificultades y costos de la opción de

20 "Ello incluye mecanismos que permitan acceder a los servicios de entidades ya existentes, como el INTA, las universidades u otras" (Rimisp, 2006: 77). 
generar tecnologías para la agricultura familiar. Por las características de este sector, cada problema tecnológico resuelto es apenas un caso particular difícilmente generalizable; pero, además, están los limitantes -también estructurales- de las reales alternativas tecnológicas disponibles en el marco del contexto socioeconómico y político-institucional vigente. Dicho de otro modo, la tecnología no es una variable independiente del patrón productivo vigente $y$, por tanto, los grados de libertad para modificarla están acotados.

En este sentido, "la cuestión es política y no tecnológica" (Da Silva, 1999: 174); para que la política agrícola y tecnológica (precios, créditos, I\&D, transferencia, etc.) dirigida a este sector sea efectiva, es preciso asegurar legal e institucionalmente, por mecanismos democráticos, las posibilidades de contrabalancear su escaso "poder económico real con el expresivo peso político que potencialmente poseen” (Da Silva, 1999: 174)

\subsection{La dimensión local}

Básicamente, el Rimisp propone una estrategia centrada en dos pilares institucionales: la descentralización y la concertación; ello supone el alineamiento de los gobiernos locales, las organizaciones agrorrurales y, en general, otro tipo de dinámicas e instituciones que operan en los territorios rurales.

La visión de un papel más prominente de los gobiernos locales no es novedosa; se ha instalado a través de las distintas vertientes teóricas del desarrollo local y, particularmente, el desarrollo endógeno. Entre lo que debería ser y lo que es se plantea, sin embargo, una gran distancia, sobre todo cuando se trata de los estados locales en escenarios periféricos. Hilhorst (1997: 19) sistematiza los problemas más comunes a partir de evidencias en centros secundarios, concluyendo en dos aspectos que aquí interesa resaltar:

- Los bajos niveles de desarrollo económico limitan las posibilidades de una descentralización efectiva a los gobiernos locales. En general, las habilidades del gobierno local difieren según el tamaño de la población del centro en el cual ejercen la autoridad.

- Lo anterior se traduce en la presencia de otro círculo vicioso del desarrollo espacial. "Al tiempo que, en el argumento de Myrdal, sólo los recursos privados tienden a 
mover hacia adelante un número restringido de puntos en el espacio, las diferencias existentes entre las autoridades locales tenderán a ocasionar flujos de recursos públicos en la misma dirección, reforzándose mutuamente y tendiendo por lo tanto a fortalecer las estructuras espaciales existentes" (Hilhorst, 1997: 20).

Cabe advertir, a su vez, que los objetivos de las políticas rurales pueden enfrentarse a los de otras políticas sectoriales o macroeconómicas aplicadas simultáneamente (políticas de importación de alimentos, cambiaria, de control de precios, entre otras). De Villalobos (2001) resalta esta contradicción como una de las razones que explica los fracasos de las políticas orientadas a reducir la pobreza rural en el ámbito local. Por su parte, Lattuada et al. advierten que las políticas centradas en lo local pueden ser significativas pero "resultan una cura efímera o una porción homeopática para unos pocos, si las condiciones estructurales y la política económica y sectorial resultan adversas o neutras" (2006: 173).

\section{A modo de conclusión: problemas y desafíos de las políticas rurales}

El rumbo adoptado por el crecimiento de la economía agrorrural de la provincia exhibe, al igual que en el país, la incapacidad para atender los problemas de la exclusión y la agudización de las desigualdades. Las herramientas de intervención implementadas no han dado solución a los sectores de la agricultura familiar que quedaron fuera de la actividad agropecuaria y/o del mercado de trabajo. Visto desde la categoría de pequeña producción agropecuaria (PPA), de las más de 27,000 EAPs registradas en la provincia por el Censo Nacional Agropecuario (2002), 24\% corresponde al estrato de las más pobres y vulnerables, que muy probablemente no han perdido su condición por la incidencia de los programas asistenciales.

Por su parte, hay serios problemas en aquellos territorios cuya funcionalidad, ligada a la base agropecuaria, está fuertemente tensionada por las lógicas globales-sistémicas que atraviesan a estas producciones. Una rápida mirada a la estructura rural-urbana provincial revela que hay más de cien localidades rurales en riesgo de desaparición y alrededor de 18 partidos rurales que han perdido población en la última década. 
El desafío planteado es cómo construir un nuevo entorno de políticas activas desde una redefinición conceptual de la visión asistencial, focalizada en la pobreza rural, que predomina desde el inicio de los años noventa.

Una primera cuestión a resolver es, entonces, la del enfoque global de la estrategia. Si se admite que el desarrollo rural es mucho más que una cuestión de los pobres, cabe contemplar la atención de las necesidades básicas de los habitantes rurales de la provincia, lo cual no admite discusión en un marco de políticas de mediano y largo plazos que fortalezcan posibilidades de transformación económica, social, ambiental y política del medio agrorrural. Estas políticas deberían tener en cuenta los siguientes prerrequisitos (Gorenstein et al., 2006):

- La necesaria inserción en un enfoque global de políticas macroeconómica, sectorial, territorial y de desarrollo rural.

- Que las políticas rural y agroalimentaria se conciban como capítulos de la estrategia de desarrollo territorial, en tanto los complejos agroalimentarios (CAA) en sí mismos son inseparables del ámbito territorial (rural y urbano) de interacción cotidiana.

- Una nueva metodología y estilo de hacer política agrorrural y, en un sentido más general, territorial, articulando diversas dimensiones, planos y niveles (tramas o CAA, localregional, municipios-regiones de alta ruralidad, zonas o regiones agroproductivas, sistemas o redes de innovación locales, por citar algunos ejemplos). Asimismo, dos cuestiones clave que debemos considerar: 1) los peligros de una excesiva descentralización en el ámbito local, la jerarquización teórica y normativa del rol de los gobiernos locales no debe dar lugar a transferencias generalizadas, y sin la creación de las condiciones, capacidades y competencias requeridas. Las políticas descentralizadas suponen complejidad de contenidos y diferentes alcances abarcando, entre otras, distintas escalas de organización social y territorial y 2) la importancia de la flexibilidad de ciertos programas para ajustar y adecuar su ejecución a cambios de contexto (macroeconómicos, sectoriales, territoriales, etcétera). 


\section{Bibliografía}

Abramovay, Ricardo (2006), "Para una teoría de los estudios territoriales", en Mabel Manzanal, Guillermo Neiman y Mario Lattuada (comps.), Desarrollo rural. Organizaciones, instituciones y territorios, Ciccus, Buenos Aires, Argentina, pp. 51-70.

BID (Banco Interamericano de Desarrollo) (2005), "Perfil avanzado. Política de desarrollo rural", documento de circulación, www.bid.org.

Basualdo, Eduardo y Miguel Khavisse (1993), El nuevo poder terrateniente, Planeta, Buenos Aires, Argentina.

Benencia, Roberto y Germán Quaranta (2005), "Producción, trabajo y nacionalidad: configuraciones territoriales de la producción hortícola del cinturón verde bonaerense", Revista Interdisciplinaria de Estudios Agrarios, 23, Universidad de Buenos Aires, Buenos Aires, Argentina, pp. 101-132.

Carballo, Carlos (2001), "Nueva institucionalidad para el desarrollo rural en Argentina", ponencia presentada en II Jornadas Interdisciplinarias de Estudios Agrarios y Agroindustriales, Programa Interdisciplinario de Estudios Agrarios, Facultad de Economía de la Universidad de Buenos Aires, Buenos Aires, Argentina.

De Janvry, Alain y Elisabeth Sadoulet (2001), "La inversión en desarrollo rural es buen negocio", en R. Echeverría (ed.), Desarrollo de las economías rurales, Banco Interamericano de Desarrollo, Washington, pp. 1-42.

Etxezarreta, Miren (2003), "Las políticas de desarrollo rural integrado y la agricultura familiar”, Universidad Autónoma de Barcelona, Barcelona, España.

Gorenstein Dejter, Silvia Mirta, Andrea Cecilia Barbero de Cos, Martín Napal y Mariana Olea (2006), "Diagnóstico sobre la situación agraria y rural de la provincia de Buenos Aires. Orientaciones para una estrategia de desarrollo rural", Documento Proinder, Secretaría de Agricultura, Ganadería, Pesca y Alimentos (SAgrya)-Ministerio de Asuntos Agrarios 
de la Provincia de Buenos Aires, Buenos Aires-La Plata, Argentina.

Hilhorst, Jean (1997), “Desarrollo local/regional e industrialización”, EURE, XXII(68), Santiago de Chile, p. 19.

INDEC (Instituto Nacional de Estadisticas Económicas), “Censo Nacional Agropecuario”, 2002, www.indec.gov.arfg.

Lattuada, Mario, Juan Mauricio Renold, Luciana Binolfi y Adriana de Biasi (2006), "Limitantes al desarrollo territorial rural en contextos de políticas sectoriales neutras o negativas", en Mabel Manzanal, Guillermo Neiman y Mario Lattuada, Desarrollo rural. Organizaciones, instituciones y territorios, Ciccus, Buenos Aires, Argentina, pp. 153-175.

Manzanal, Mabel (2006), "Regiones, territorios e institucionalidad del desarrollo rural", en Mabel Manzanal, Guillermo Neiman y Mario Lattuada, Desarrollo rural. Organizaciones, instituciones y territorios, Ciccus, Buenos Aires, Argentina, pp. 21-50.

Manzanal, Mabel (2005), "Modelos de intervención de los proyectos de desarrollo rural en Argentina a partir de 1995", Rimisp-SAGPyA, www.rimisp.org.

Neiman, Guillermo y Mario Lattuada (2005), El campo argentino. Crecimiento con exclusión, Capital Intelectual, Buenos Aires, Argentina.

PROINDER-IICA (2006), Los pequeños productores en la República Argentina. Importancia de la producción agropecuaria y el empleo en base al Censo Nacional Agropecuario 2002, PROINDER-IICA, Buenos Aires, Argentina.

Rossi, Carlos y Carlos León (2005), “Temas fundamentales en la inserción de pequeños productores en cadenas comerciales para una estrategia de desarrollo rural", documento del proyecto Rimisp-sagpya, www.rimisp.org.

Salama, Pierre (2006), "La apertura revisada: crítica teórica y empírica al libre comercio", Cuadernos del CENDES, año

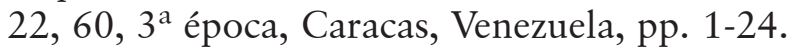


Silva, José Graziano da (1999), Tecnología e agricultura familiar, Editora de Universidade Federal do Rio Grande do Sul, Rio Grande do Sul, Brasil.

Slutzky, Daniel (2003), "A propósito del Censo Nacional Agropecuario 2002", Realidad Económica, 196, IADE, Buenos Aires, Argentina, pp. 77-83.

Soverna, Susana (2004), "Políticas de desarrollo rural: situación actual y propuestas", ponencia presentada en el II Congreso Nacional de Políticas Sociales, 15-17 de septiembre, Mendoza.

Rimisp-Centro Latinoamericano para el Desarrollo Rural (2006), "Propuesta para una estrategia de desarrollo rural de la Argentina”, www.rimisp.org.

Rodríguez, Juan y Nicolás Arceo (2006), “Renta agraria y ganancias extraordinarias en la Argentina 1990-2003", Realidad Económica, 219, IADE, Buenos Aires, Argentina, pp. 76-98.

Teubal, Miguel (2006), "Expansión del modelo sojero en la Argentina. De la producción de alimentos a los commodities", Realidad Económica, 220, IADE, Buenos Aires, Argentina, pp. 71-96.

Tsakoumagkos, Pedro, Susana Soverna y Clara Craviotti (2002), Campesinos y pequeños productores en las regiones agroeconómicas de Argentina, PROINDER, Buenos Aires, Argentina.

Villalobos, Ruby de (2001), "Desarrollo rural y desarrollo local: descentralización y participación democrática como requisito para una efectiva lucha contra la pobreza rural", en David Burin y Ana Inés Heras (comps.), Desarrollo local. Una respuesta a escala humana a la globalización, Ciccus-La Crujía, Buenos Aires, Argentina, pp. 187-234.

Recibido: 30 de agosto de 2007. Aceptado: 4 de marzo de 2008. 
Silvia Gorenstein. Es licenciada y magíster en economía por el Departamento de Economía de la Universidad Nacional del Sur; realizó estudios de especialización en planificación regional; posgrado en desarrollo económico y planificación en el Instituto Latinoamericano de Planificación Económica y Social (ILPES-Comisión Económica para América Latina) en Santiago de Chile. Actualmente es investigadora independiente del Consejo Nacional de Investigaciones Científicas y Técnicas (Conicet); docente-investigadora categoría I en el Programa de Incentivos Universidades Nacionales; profesora asociada en la cátedra economía regional de la licenciatura en economía del Departamento de Economía de la Universidad Nacional del Sur. Es directora de la maestría en desarrollo y gestión territorial (ciclo Universidad Nacional del Sur) y profesora invitada en programas de posgrado en Argentina y otros países. Sus líneas de investigación son: dinámicas innovadoras e institucionales en tramas agroalimentarias de la región pampeana, incluidas sus implicaciones y lógicas territoriales. Entre sus publicaciones recientes destacan: "Dinámicas en una trama hortícola y efectos territoriales. El caso del valle bonaerense del Río Colorado", Revista Interdisciplinaria de Estudios Agrarios, 24, Buenos Aires, pp. 81-99 (2006); "Nueva institucionalidad y proyección local tras la privatización portuaria argentina. Los casos de Bahía Blanca y Rosario", EURE, XXXI(92), Pontificia Universidad Católica de Chile, Santiago, pp. 105-123 (2005); "Caracteristiques territoriales des changements du systeme agroalimentaire de la pampa", Études Rurales, 165-166, EHESS, París, pp. 147-169 (2003); “Territorio y sistemas agroalimentarios, enfoques conceptuales, dinámicas recientes en Argentina", Desarrollo Económico, 43(168), IDES, Buenos Aires, pp. 563587 (2003); es compiladora, en coautoría, del libro Complejos productivos basados en recursos naturales y desarrollo territorial. Estudios de caso en Argentina, Universidad Nacional del Sur, Bahía Blanca (2006).

Martín Napal. Es licenciado en economía por la Universidad Nacional del Sur; actualmente cursa la maestría en desarrollo y gestión territorial en la misma institución. Es investigador del proyecto "Innovación, instituciones y governance en tramas agroalimentarias del sur pampeano" y está elaborando su tesis de magíster en el marco de temáticas relacionadas con sectores sociales locales de alta vulnerabilidad. Es funcionario del gobierno municipal de la ciudad de Bahía Blanca y director del Programa 
de Empleo Municipal. Entre sus publicaciones destaca: "Pymes industriales del sudoeste de la provincia de Buenos Aires: diagnóstico de situación y posibilidades de inserción en el Mercosur", Informe de Coyuntura, año 9, 78, Centro de Estudios Bonaerenses, La Plata, pp. 54-79 (1999).

Andrea Barbero. Es licenciada y magíster en economía por la Universidad Nacional del Sur. Actualmente se desempeña como profesora adjunta y secretaria académica del Departamento de Economía en la misma institución. Su línea de investigación se vincula al estudio de los procesos de innovación y cambios institucionales y sus efectos sobre las tramas agroalimentarias del sur pampeano. Integrando el proyecto de investigación "Sistemas agroalimentarios y nuevas formas de articulación territorial", del Conicet. Algunas de sus publicaciones son: "Nuevos actores y dinámicas territoriales en el complejo oleaginoso del sur de la provincia de Buenos Aires”, en J. Barbosa Cavalcanti y G. Neiman (comps.), Acerca de la globalización en la agricultura. Territorios, empresas y desarrollo local en América Latina, Ciccus, Buenos Aires, pp. 228-250 (2006); en coautoría, "Dinámicas de innovación y capacidades localizadas en tramas agroalimentarias pampeanas", en A. C. Torres Ribeiro, H. Tavares, J. Natal y R. Piquet (eds.), Globalización y territorio. Ajustes periféricos, Arquímedes Ediciones-IPpur, Río de Janeiro, pp. 437-464 (2005); en coautoría, "Cambios institucionales en el complejo triguero argentino. Algunas consideraciones sobre la región del sudoeste bonaerense", en María del Carmen Vaquero (comp.), Territorio, economía y medio ambiente en el sudoeste bonaerense, Ediuns, Bahía Blanca, pp. 267-290 (2002). 